\title{
CONVIVÊNCIA COM FILHOS COM \\ TRANSTORNO DO ESPECTRO AUTISTA: \\ DESVELANDO SENTIDOS DO SER-AÍ-MÃE
}

\author{
LIVING WITH CHILDREN WITH AUTISTIC \\ SPECTRUM DISORDER: UNVEILING \\ THE MEANINGS OF THE BEING-THERE-MOTHER
}

\section{CONVIVIENDO CON NIÑOS CON EL TRASTORNO DEL ESPECTRO AUTISTA: DESVELLANDO LOS SENTIDOS DEL SER-AHÍ-MADRE}

\author{
Daniela de Cássia Sabará Rendon ${ }^{1}$ \\ Anna Maria de Oliveira Salimena ${ }^{2}$ \\ Thaís Vasconselos Amorim ${ }^{3}$ \\ Andyara do Carmo Pinto Coelho Paiva ${ }^{3}$ \\ Maria Carmen Simões Cardoso de $\mathrm{Melo}^{4}$ \\ Bárbara Lacy Vitorino Batista ${ }^{5}$
}

Como citar este artigo: Rendon DCS, Salimena AMO, Amorim TV, Paiva ACPC, Melo MCSC, Batista BLV. Convivência com filhos com transtorno do espectro autista: desvelando sentidos do ser-aí-mãe. Rev baiana enferm. 2019;33:e31963.

Objetivo: desvelar sentidos de mães na convivência com filhos acometidos pelo transtorno de espectro autista (TEA). Método: pesquisa qualitativa, ancorada na fenomenologia heideggeriana, com dados coletados por meio de entrevista fenomenológica com 14 mulheres/mães, entre janeiro e março de 2016. Resultados: as mães significaram que a convivência trazia aprendizado, mudanças como ser humano e busca por saber tudo a respeito de autismo; e não conseguir trabalhar, por ter de cuidar do filho, sentir-se excluída e sobrecarregada. Conclusão: o alcance do movimento existencial do ser-aí-mãe na convivência com o filho acometido pelo TEA permitiu compreender as mudanças, exigências e cobranças que cabiam à mulher que, de modo inautêntico, ocupava-se com a rotina de cuidados ao filho. Preocupando-se em oferecer o melhor de si, sentia-se distante do cuidado consigo e com suas relações sociais e familiares. Do mesmo modo, pela fragilidade da rede de apoio social, tornava-se vulnerável biopsicosocioespiritualmente.

Descritores: Transtorno Autístico. Relações Mãe-Filho. Cuidados de Enfermagem.

Objective: unveiling the meanings of mothers who coexist with children affected by the autism spectrum disorder (ASD). Method: qualitative research based on Heideggerian phenomenology, with data collected through a phenomenological interview with 14 women/mothers, from January to March 2016. Results: the mothers signified that the coexistence led to learning, changes as human beings, and the search to know everything about autism; as

\footnotetext{
Enfermeira. Pesquisadora independente. Mestre em Enfermagem. Juiz de Fora, Minas Gerais, Brasil.

Enfermeira. Doutora em Enfermagem. Professora Titular da Universidade Federal de Juiz de Fora. Juiz de Fora, Minas Gerais, Brasil.

Enfermeira. Doutora em Enfermagem. Professora Adjunta da Universidade Federal de Juiz de Fora. Juiz de Fora, Minas Gerais, Brasil. thaisamorim80@gmail.com

Enfermeira. Doutora em Enfermagem. Professora Associada da Universidade Federal de Juiz de Fora. Juiz de Fora, Minas Gerais, Brasil.
}

Estudante de Enfermagem. Universidade Federal de Juiz de Fora. Juiz de Fora, Minas Gerais, Brasil. 
well as not being able to work due to the care the child requires, feeling excluded and overloaded. Conclusion: the reach of the existential movement of the being-there-mother in the coexistence with the son affected by ASD allowed to understand changes, demands, and burdens on women who, inauthentically, were engaged in the routine of care of their children. Worried with the fact they should offer all they could, they felt distant from the care with themselves and from their social and family relations. Similarly, due to the fragility of their social support network, they became bio-psycho-social-spiritually vulnerable.

Descriptors: Autistic Disorder. Mother-Child Relations Nursing Care.

Objetivo: desvelar los sentidos de las madres que conviven con niños acometidos por el trastorno del espectro autista (TEA). Método: investigación cualitativa, basada en la fenomenología Heideggeriana, con datos colectados por medio de una entrevista fenomenológica con 14 mujeres/madres, de enero a marzo de 2016. Resultados: madres significaron que convivencia traía aprendizaje, cambios en los modos de cuidar del niño, sentimientos de exclusión y sobrecarga. Conclusión: el alcance del movimiento existencial del ser-abi-madre en la convivencia con el bijo acometido por el TEA permitió comprender los cambios, exigencias y demandas sobre la mujer que, de manera inauténtica, se ocupaba con la rutina de cuidados a su bijo. Se preocupando en ofrecer su mejor, sentíase distante del cuidado con ella misma y de sus relaciones sociales y familiares. De la misma manera, debido a la fragilidad de su red de apoyo social, se volvía biopsicosocioespiritualmente vulnerable.

Descriptores: Trastorno Autístico. Relaciones Madre-Hijo. Atención de Enfermería.

\section{Introdução}

O transtorno do espectro autista (TEA) é uma síndrome que interfere na condição do neurodesenvolvimento, com manifestações que se mostram já nos primeiros anos de vida, como deficiência persistente total ou parcial da comunicação e interação sociais, padrões de comportamento restrito e/ou repetitivos e grande interesse por objetos ou atividades específicas. Pessoas acometidas por esse transtorno apresentam habilidades cognitivas bem variáveis, que vão desde o retardo mental até capacidades superiores à média populacional não acometida. O curso do TEA é crônico e a incapacidade funcional na idade adulta resulta diretamente da ineficácia do acompanhamento e tratamento multiprofissional ao longo da vida ${ }^{(1)}$.

Apesar de não haver um consenso sobre os fatores causais que culminam no desenvolvimento do transtorno, pesquisas em âmbito mundial sinalizam o aumento da prevalência e incidência de TEA nas duas últimas décadas, sendo de 1 para cada 59 crianças em $2018^{(2-3)}$. Ao considerar esses dados, torna-se relevante voltar a atenção à família dessas pessoas, especialmente à figura materna, uma vez que o cuidar é uma ação culturalmente delegada à mulher. No cotidiano, crianças, adolescentes ou adultos com TEA demandam necessidades de atenção e cuidado específicas por parte de suas mães, as quais se sentem sobrecarregadas física e emocionalmente $^{(4)}$.

As mães revelam que dificuldades quanto à inserção de seus filhos no meio familiar, escolar e social desencadeiam tristeza, estafa mental, distanciamento de amigos, familiares e falta de tempo para dedicação pessoal. Para além, evidencia-se a insatisfação e o estresse pela insuficiência de orientação sobre o transtorno e o acesso a tratamento e terapias infantis. Atrelado a isso, surgem desafios de ordem emocional e econômica, fazendo com que as mulheres cuidem de si e de seus filhos de modo, por vezes, ineficaz ${ }^{(5-7)}$.

Cabe ponderar acerca da vulnerabilidade programática a que a mulher está sujeita, pela fragilidade da rede de apoio junto ao binômio mãe/filho ${ }^{(5,8)}$. Sabe-se que uma rede estável, com programas e políticas de saúde bem estabelecidos, viabilizaria o cuidado integral, longitudinal e coordenado preconizado pelo Sistema Único de Saúde (SUS). Para tal, deve-se investir na qualificação dos profissionais de saúde, com enfoque na atenção biopsicosocioespiritual para com as pessoas acometidas pelo transtorno, 
abrangendo as várias disciplinas envolvidas no campo da pesquisa e da assistência, que subsidiem orientação e capacitação maternas ${ }^{(9-11)}$.

Nessa perspectiva, destaca-se a Enfermagem como profissão que tem por essência o cuidado ao Ser em situação de autismo e aos seus familiares. Desde a formação, o enfermeiro é incitado e habilitado para desenvolver competências técnicas e humanísticas, por meio de ações conscientes de cuidado, as quais abrangem acolhimento, vínculo, capacidade de decisão, sensibilidade e pensamento crítico ${ }^{(12-13)}$.

Relativamente ao TEA, o enfermeiro pode colaborar para a elucidação do diagnóstico, por meio da observação comportamental da criança nas consultas de enfermagem, visitas domiciliares e internação hospitalar ${ }^{(14)}$. Ademais, ouvindo e considerando as observações da família, é oportunizada a troca de saberes a respeito do transtorno, o acompanhamento e a avaliação do grau de compreensão dos membros envolvidos, além do oferecimento de apoio e cuidado ao familiar diante da realidade que se apresenta ${ }^{(15)}$. Entretanto, no tocante ao atendimento à mãe como cuidadora principal, é relevante avançar nas perspectivas de compreensão da mulher-mãe que vivencia junto ao filho o TEA, possibilitando parceria no cuidado e manejo das situações para além do modelo biomédico ${ }^{(12,16-17)}$.

Assim, pela crescente epidemiologia do TEA e pela importância de que se reveste no plano do cuidado integral à pessoa, família e coletividade, o objetivo deste artigo é desvelar sentidos de mães na convivência com filhos acometidos pelo transtorno de espectro autista.

\section{Método}

Pesquisa qualitativa fundamentada na fenomenologia pensada por Martin Heidegger, a qual possibilita a compreensão da essência humana em sua existência, que se mostra como possibilidades de abertura e movimento ${ }^{(18)}$. Utilizou-se o Consolidated Criteria for Reporting Qualitative Research (COREQ) como diretriz para descrição metodológica.
A fim de atender ao objetivo proposto, acessaram-se as possíveis participantes em reuniões organizadas por um grupo independente de familiares de pessoas acometidas pelo TEA, em uma cidade da Zona da Mata Mineira. Os critérios de inclusão compreenderam: ser mulher, maior de 18 anos e mãe de filho(a) com diagnóstico de transtorno de espectro autista; os de exclusão: aquela que não estivesse em condições emocionais para se expressar.

Por meio da amostragem intencional e da apresentação da proposição de pesquisa em uma das reuniões do grupo, houve o convite à participação de mulheres/mães, com aquiescência de 14 dentre elas. A etapa de campo ocorreu entre janeiro e março de 2016, por meio da entrevista fenomenológica ${ }^{(19)}$, sendo 10 realizadas em domicílio e 4 em local público, por preferência das participantes. Destaca-se que o local público foi em área situada próximo à sede, onde as mulheres levavam seus filhos para acompanhamento. Em todas as entrevistas, buscou-se preparar o local, de modo a proporcionar um ambiente calmo e adequado o suficiente para possibilitar o desenvolvimento de genuínos encontros fenomenológicos, com privacidade para o discorrer dos depoimentos, após assinatura do Termo de Consentimento Livre e Esclarecido.

As entrevistas foram gravadas em áudio e tiveram duração média de 20 minutos. A fim de garantir o anonimato, as participantes foram identificadas por meio da letra T seguida do número correspondente à ordem da entrevista (T1, $\mathrm{T} 2, \mathrm{~T} 3, \ldots, \mathrm{T} 14)$.

Os instrumentos de coleta de dados utilizados foram o roteiro de entrevista, composto de perguntas relacionadas à historiografia das participantes (estado civil, número de filhos, número de filhos com TEA, data de nascimento e de diagnóstico dos filhos com TEA, religião, escolaridade, profissão) e de questão orientadora da entrevista (Como é para você o dia a dia com o diagnóstico de autismo de seu filho?), além do diário de campo, que permitiu o registro da comunicação não verbal das participantes, expressa em gestos e/ou outras manifestações. 
Após transcrição, leituras e escutas atentivas dos conteúdos das entrevistas, a análise pautada no referencial de Heidegger iniciou-se pela evidência das estruturas essenciais e construção das unidades de significação: A convivência traz aprendizado, mudanças como ser humano e busca por saber tudo a respeito de autismo; e não conseguir trabalhar, por ter de cuidar do filho, sentir-se excluída e sobrecarregada.

No movimento analítico hermenêutico, buscou-se a compreensão dos fatos pelos significados expressos na esfera ôntica, ou de compreensão vaga e mediana, e posterior desvelamento dos sentidos na esfera ontológica, ou de compreensão interpretativa.

O encerramento da etapa de coleta de dados ocorreu mediante análise em profundidade dos textos constituintes das entrevistas, nos quais não se observou distinções que implicassem em novos depoimentos. Ademais, considera-se que o fenômeno em estudo - mães na convivência com filhos acometidos pelo transtorno de espectro autista - foi alcançado por meio da suficiência de significados que atenderam ao objetivo proposto.

Os aspectos éticos foram observados e foram cumpridas todas as recomendações da Resolução n ${ }^{\circ}$ 466/12 do Conselho Nacional de Saúde. A aprovação e o deferimento pelo Comitê de Ética em Pesquisa da Universidade Federal de Juiz de Fora apresentaram-se sob o Parecer número 1.254.423, CAAE 49453115.5.0000.5147.

\section{Resultados}

A média de idade das 14 participantes foi de 39,9 anos. Sobre o estado civil, 57,14\% eram casadas, 35,7\% solteiras e 7,14\% divorciadas. A média de filhos por mulher foi de 1,8 e 100\% tinha apenas um filho com TEA. A média da idade do filho no momento do diagnóstico foi de 2,6 anos. Dos filhos acometidos pelo transtorno, 10 eram do sexo masculino e 4 do sexo feminino. Sobre a escolaridade das mães, 64,3\% possuíam ensino médio completo, 7,1\% ensino superior incompleto e $28,6 \%$, superior completo. Sobre a ocupação, 7 declararam-se donas de casa.
Em "A convivência traz aprendizado, mudanças como ser humano e busca por saber tudo a respeito de autismo", a vivência de mães com o diagnóstico de transtorno de espectro autista significou aprender mais com o filho do que este com elas na convivência. Nesse aprendizado, mudaram como seres humanos, passaram a fazer coisas que antes não conseguiam e a esperar o tempo do filho, conforme expressam os textos:

\begin{abstract}
A convivência com G. me tem feito aprender [...] Você vai aprendendo que tinha que ser você. Que eu precisava me lapidar enquanto ser humano [...] Até o meu tom de voz, pra conversar hoje [...] é diferente. (T2).
\end{abstract}

Depois que eu tive meu filbo, eu sei o que é ser humano [...] eu aprendo muito com S., porque eu descobri uma força que eu não sabia que eu tinha! O S. me fez enxergar que eu não era aquela fracassada [...] que vivia chorando, sabe? (T13).

No caso do autismo, a gente que tem que mudar a nossa forma de ser e de agir [...] hoje eu já me sinto uma pessoa muito feliz pela filha que eu tenho. A gente aprende muito com eles. A gente tem mais a aprender com eles do que eles com a gente. (T3).

A gente acostuma, aprende a conviver, entra no mundo deles e tenta trazer eles para o mundo da gente. Ela não tem problema de audição, é só mesmo a fala. Então, eu aprendi a esperar esse tempo dela. (T8).

Todo dia é um aprendizado. Falo que eu sou feliz, porque tenho um autista em casa. Me levou a fazer coisas que eu não conseguia! Depois que ele entrou na APAE, foi que aprendi a conviver com ele. (T10).

Fui convivendo com ela e aprendendo também as coisas com ela [...] É porque elas nos mostram outro mundo [...] diferente desse que a gente vive, de preconceito. (T4).

As mães compreendiam que, diante de alguns aspectos do autismo, foi preciso pesquisar, participar de congressos e outros eventos, para atuar junto aos filhos e ajudar outras crianças, segundo denotam os textos:

Tive que prestar mais atenção nesse aspecto da personalidade do meu filho, nas coisas que ele estava apresentando, e eu precisava atuar nisso e, desde então, eu tenho pesquisado e participado de várias reuniões, congressos, tudo a respeito de autismo. E isso tem sido muito bom. (T5).

Aprendi que, no autismo, com a quantidade de estímulos e informações que eles recebem, às vezes, eles precisam de um tempo pra trabalhar, e esse tempo para a gente é diferente. Às vezes, ele precisa recuar no processo, porque, para ele, tudo é mais lento. (T6).

Nunca tinha nem ouvido o termo autista. Ai eu comecei a procurar em internet, livro. Já participei de um monte de seminário [...] Agora, tudo o que sai de novidade de autismo, procuro saber. (T9). 
Muitas coisas que me ajudou até na minha profissão [...] porque muita coisa de fonoaudiologia [...] eu aplicava na minha escola e também isso me permitiu ajudar as crianças. (T7).

Em "Não conseguir trabalhar por ter de cuidar do filho, sentir-se excluída e sobrecarregada”, as mães significaram que a convivência com o filho dificulta ter um trabalho, ser convidada para eventos familiares e sociais, além de gerar sentimentos de falta de acolhimento, como se evidencia nos fragmentos a seguir:

[...] eu já tentei várias coisas para ter uma renda, mas às vezes é difícil conciliar o borário. (T12).

Eu parei de trabalhar, parei de fazer tudo o que eu tinha que fazer só para poder ficar com ela. (T4).

É uma sobrecarga muito grande, e eu preciso, como mãe de autista, fazer um tratamento psicológico também, até para poder lidar com essa situação [...] não existem profissionais que estão preparados para cuidar da família com essa sindrome, né? (T3).

Eu falo que eu já não tenho mais vida [...] eu estava trabalbando em pleno vapor, cuidando dos meninos e no trabalbo [...] aí, nesse ritmo todo, eu pirei [...] eu não tive esse acolbimento, sabe? Então foi muito dolorido para mim [...] me senti muito sozinha e às vezes até os amigos me acabaram deixando de fora [...] muitas vezes eu pedi ajuda, gritei: "socorro, socorro gente, eu estou aqui!"(T6).

As pessoas não convidam para festas, não convidam para nada, porque vai dar trabalho lá [...] a própria família às vezes exclui muito, porque, se você vai na casa, está incomodando. (T10).

A hermenêutica permitiu desvelar o movimento existencial do ser-aí-mãe na convivência com filho acometido pelo TEA, que se mostrou de modo inautêntico, ao descrever mudanças ditadas pela facticidade do transtorno. Desvelou-se na ocupação dos cuidados em função do transtorno e na preocupação caracterizada como solicitude junto ao filho e como deficiente junto aos amigos e familiares.

\section{Discussão}

A convivência com o filho atingido pelo TEA no presente estudo trouxe, para o ser-aí-mãe, aprendizados e descobertas ditados pelos limites e possibilidades em face do transtorno, gerando transformações internas e externas. Ao significar mudanças como ser humano, não o fez com base em si mesma, mas na condição que o TEA impõe, caracterizando o sentido da inautenticidade ${ }^{(18)}$.

De modo semelhante, mães relataram lidar bem com o novo modo de viver em função do diagnóstico de TEA do filho, apesar de sinalizarem que percebiam suas vidas como fora do normal. Isso porque, com a nova rotina de cuidados, era necessário, além do cumprimento das tarefas típicas da maternidade, um tempo maior de dedicação à rotina de cuidados diários e terapias a que o filho era submetido ${ }^{(6)}$. No momento do diagnóstico, mães e pais conseguiam assimilar e aceitar a informação de maneira equivalente, porém, desde então, a qualidade de vida materna era menor ${ }^{(20)}$, pois as dificuldades e a sobrecarga de responsabilidades e cuidados passavam a prevalecer para ela ${ }^{(10)}$.

Desvelando-se na ocupação e na preocupação caracterizada como solicitude junto ao filho, as mães deste estudo descreveram ter de se dedicar por completo aos filhos, buscar aprender e se informar sobre o TEA por meios diversos, nos quais não se observou a presença do profissional de saúde acolhendo as suas necessidades e orientando acerca dos cuidados. Esse movimento de distanciamento também ocorreu por parte de familiares, afetando as dimensões sociais e emocionais das participantes.

A mãe como cuidadora principal do filho com TEA mantém e adapta seus papéis de esposa, familiar, profissional e mulher, lidando com as consequências emocionais próprias e dos familiares ${ }^{(6)}$. A natureza prolongada desse estresse parental tem determinado significativos efeitos a curto e longo prazo na saúde mental, psicológica e física, podendo afetar negativamente o trabalho, a vida doméstica, o autocuidado e o relacionamento com outros membros da família ${ }^{(21)}$.

O apoio social é um fator de efeito positivo no enfrentamento das famílias de pessoas com TEA, ajudando a reduzir o estresse. Contudo, evidências mostram baixo apoio social constatado $^{(20)}$. As mães percebiam que passavam a ser excluídas de reuniões, encontros e conversas, ao contrário do que comumente observavam ocorrer entre amigos com filhos de idades parecidas e 
que não possuíam TEA. Ao procurarem apoio e alguém que as acolhesse em suas singularidades, só o encontravam em outras mães em situações semelhantes $^{(6)}$ ou em grupos de pais lotados em instituição especializada, que proporcionava espaço de escuta empática, troca de afetos, dúvidas e apoio profissional ${ }^{(22)}$.

O sentido da preocupação no modo deficiente indica "[...] o ser por um outro, contra um outro, sem os outros, o passar ao lado um do outro e não sentir-se tocado pelos outros"(19:178). O absenteísmo de familiares e profissionais foi assimilado pelas mães como falta de acolhimento, o que possibilita refletir acerca da ausência de mobilização, especialmente na área da saúde - em especial da enfermagem - de princípios humanísticos e de humanização, os quais envolvem escuta ativa e qualificada, responsabilização e comprometimento com as demandas do outro ${ }^{(23)}$.

A invisibilidade do enfermeiro nas significações deste estudo atesta a necessária sensibilização, mobilização e capacitação profissional nos espaços de cuidado à saúde de mães e de familiares que convivem diretamente com pessoas com TEA. No presente estudo, nota-se que o princípio da integralidade não foi alcançado pela rede de cuidados à saúde, produzindo uma vulnerabilidade programática e social ${ }^{(8,24)}$.

Com acesso a serviços mínimos para seus filhos e quase nulo para seu próprio cuidado, as mães podem desenvolver depressão e ansiedade. O pronto acesso às ferramentas de educação, terapias e orientações nesta direção torna-se basilar para potencializar a diretriz materna e familiar, a fim de promover a autonomia possível à criança com TEA e o empoderamento psicossocial da mulher. Como educador em saúde, o enfermeiro capacitado pode tornar-se o diferencial no desenvolvimento da relação mãe-filho, propiciando conhecimento sobre o transtorno e oferecendo apoio e cuidado à mãe em sua dinâmica familiar, com vistas ao cuidado integral de todos os membros envolvidos ${ }^{(15-16,21)}$.

Em se tratando de pesquisa desenvolvida sob abordagem fenomenológica, considera-se como limitação o cenário único. Todavia, acredita-se que, em vista do rigor epistemológico adotado na investigação, os resultados alcançados evidenciam relevantes aspectos a serem considerados na prática clínica de enfermeiros que atuam junto às mães e aos familiares de crianças com transtorno do espectro autista.

\section{Conclusão}

O alcance do movimento existencial do ser-aí-mãe na convivência com o filho acometido pelo TEA permitiu compreender as mudanças, exigências e cobranças que cabem à mulher que, de modo inautêntico, se ocupa com a rotina de cuidados para com o filho. Preocupando-se em oferecer o melhor de si, sente-se distante do cuidado consigo e com suas relações sociais e familiares. Do mesmo modo, pela fragilidade da rede de apoio social, torna-se vulnerável biopsicossocioespiritualmente.

No tocante à invisibilidade dos profissionais de saúde, entre esses o enfermeiro, pondera-se que oportunizar mais conhecimento e domínio para além dos aspectos biológicos do transtorno, pode resultar em maior amparo e cuidado às mães. Como aliado a esse processo, destaca-se o necessário esforço em definir e efetivamente aplicar as políticas públicas de cuidados à saúde da mulher de maneira articulada às necessidades que enfrenta.

Por fim, cabe destacar que o referencial da fenomenologia heideggeriana com enfoque no Ser permite e auxilia a compreensão das subjetividades que permeiam o cotidiano da saúde, apontando direções que possibilitam iluminar novas pesquisas, ampliando o saber em Enfermagem.

\section{Colaborações:}

1 - concepção, projeto, análise e interpretação dos dados: Daniela de Cássia Sabará Rendon, Anna Maria de Oliveira Salimena e Thaís Vasconselos Amorim;

2 - redação do artigo e revisão crítica relevante do conteúdo intelectual: Daniela de Cássia Sabará Rendon, Anna Maria de Oliveira Salimena, Thaís Vasconselos Amorim, Andyara 
do Carmo Pinto Coelho Paiva, Maria Carmen Simões Cardoso de Melo e Bárbara Lacy Vitorino Batista;

3 - aprovação final da versão a ser publicada: Daniela de Cássia Sabará Rendon, Anna Maria de Oliveira Salimena, Thaís Vasconselos Amorim, Andyara do Carmo Pinto Coelho Paiva, Maria Carmen Simões Cardoso de Melo e Bárbara Lacy Vitorino Batista.

\section{Referências}

1. American Psychiatric Association. DSM-5: Manual diagnóstico e estatístico de transtornos mentais. Porto Alegre: Artmed; 2014.

2. Geier DA, King PG, Hooker BS, Dórea JG, Kern JK, Sykes LK, et al. Thimerosal: clinical, epidemiologic and biochemical studies. Clin Chim Acta. 2015;444:212-20. DOI: 10.1016/j.cca.2015.02.030

3. Baio J, Wiggins L, Christensen DL, Maenner MJ, Daniels J, Warren Z, et al. Prevalence of Autism Spectrum Disorder Among Children Aged 8 Years - Autism and Developmental Disabilities Monitoring Network, 11 Sites, United States, 2014. MMWR Surveill Summ [Internet]. 2018 [cited 2018 Dec 18];67(6):1-23. Available from: https://www. ncbi.nlm.nih.gov/pmc/articles/PMC5919599/

4. Sullivan WF, Diepstra H, Heng J, Ally S, Bradley E, Casson I, et al. Primary care of adults with intellectual and developmental disabilities. Can Fam Physician [Internet]. 2018 Apr [cited 2018 Dec 18];64(4):254-79. Available from: https://www. cfp.ca/content/cfp/64/4/254.full.pdf

5. Barbosa TA, Reis KMN, Lomba GO, Alves GV, Braga PP. Rede de apoio e apoio social às crianças com necessidades especiais de saúde. Rev Rene [Internet]. 2016 [cited 2018 Dec 18];17(1):60-6. Available from: file:///C:/ Users/Home/Downloads/2606-4735-1-SM.pdf

6. Joosten AV, Safe AP. Management strategies of mothers of school $\square$ age children with autism: Implications for practice. Aust Occupa Ther J. 2014;61(4):249-58. DOI: 10.1111/1440-1630.12116

7. Hartley SL, Schultz HM. Support needs of fathers and mothers of children and adolescents with autism spectrum disorder. J Autism Dev Disord [Internet]. 2015 [cited 2018 Dec 18];45(6):1636-48. Available from: https://www.ncbi.nlm.nih.gov/ pmc/articles/PMC4442745/pdf/nihms-645826.pdf
8. Ayres JRCM. O conceito de vulnerabilidade e as práticas de saúde: novas perspectivas e desafios. In: Czeresnia D, Freitas CM, organizadores. Promoção da saúde: conceitos, reflexões, tendências. Rio de Janeiro: Fiocruz; 2003. p. 117-39.

9. Ho HS, Yi H, Griffiths S, Chan DF, Murray S. 'Do It Yourself' in the parent-professional partnership for the assessment and diagnosis of children with autism spectrum conditions in Hong Kong: A qualitative study. Autism. 2014 [cited 2018 Dec 18];18(7):832-44. DOI: 10.1177/1362361313508230

10. Poslawsky IE, Naber FB, Van Daalen E, Van Engeland $\mathrm{H}$. Parental reaction to early diagnosis of their children's autism spectrum disorder: an exploratory study. Child Psychiatry Hum Dev. 2014;45(3):294-305.

11. Gomes PTM, Lima LHL, Bueno MKG, Araújo LA, Souza NM. Autism in Brazil: a systematic review of family challenges and coping strategies. J Pediatr [Internet]. 2015 [cited 2018 Dec 18];91(2):111-21. Available from: http://www.scielo.br/pdf/jped/ v91n2/pt_0021-7557-jped-91-02-00111.pdf

12. Salviano MEM, Nascimento PDFS, Paula MA, Vieira CS, Frison SS, Maia MA, et al. Epistemologia do cuidado de enfermagem: uma reflexão sobre suas bases. Rev bras enferm [Internet]. 2016 [cited 2019 Feb 18];69(6):1240-5. Available from: http:// www.scielo.br/pdf/reben/v69n6/0034-7167reben-69-06-1240.pdf

13. Costa PCP, Garcia APRF, Toledo VP. Acolhimento e cuidado de enfermagem: um estudo fenomenológico. Texto Contexto Enferm [Internet]. 2016 [cited 2019 Feb 18];25(1):e4550015. Availablefrom:http://www.scielo.br/pdf/tce/v25n1/ pt_0104-0707-tce-25-01-4550015.pdf

14. Conselho Federal de Enfermagem. Resolução n. 358, de 15 de outubro de 2009. Dispõe sobre a Sistematização da Assistência de Enfermagem e a implementação do Processo de Enfermagem em ambientes, públicos ou privados, em que ocorre o cuidado profissional de Enfermagem, e dá outras providências. Brasília, DF; 2009.

15. Ebert M, Lorenzini E, Silva EF. Mães de crianças com transtorno autístico: percepções e trajetórias. Rev gaúcha enferm [Internet]. 2015 [cited 2018 Dec 18];36(1):49-55. Available from: file:///C:/Users/ Home/Downloads/43623-222965-2-PB.pdf

16. Mapelli LD, Barbieri MC, Castro GVDZB, Bonelli MA, Wernet M, Dupas G. Child with autistic spectrum disorder: care from the family. Esc Anna 
Nery Rev Enferm [Internet]. 2018 [cited 2018 Dec 18];22(4):20180116. Available from: http://www. scielo.br/pdf/ean/v22n4/pt_1414-8145-ean-2204-e20180116.pdf

17. Bonis SA, Sawin KJ. Risks and protective factors for stress self-management in parents of children with autism spectrum disorder: an integrated review of the literature. J Pediatr Nurs. 2016;31(6):567-79. DOI: 10.1016/j.pedn.2016.08.006

18. Heidegger M. Ser e Tempo. Petrópolis (RJ): Vozes; 2011.

19. Paula CC, Padoin SMM, Terra MG, Souza IEO, Cabral IE. Modos de condução da entrevista em pesquisa fenomenológica: relato de experiência. Rev bras enferm [Internet]. 2014 [cited 2019 Mar 20];67(3):468-72. Available from: http:// www.scielo.br/pdf/reben/v67n3/0034-7167reben-67-03-0468.pdf

20. Kuru N, Piyal B. Perceived social support and quality of life of parents of children with Autism. Niger J Clin Pract [Internet]. 2018 [cited 2019 Mar 20];21(9):1182-9. Available from: http://www.njcponline.com/temp/ NigerJClinPract2191182-6221417_171654.pdf

21. Dardas LA, Ahmad MM. Predictors of quality of life for fathers and mothers of children with autistic disorder. Res Dev Disabil. 2014;35(6):1326-33. DOI: $10.1016 /$ j.ridd.2014.03.009

22. Zanatta EA, Menegazzo E, Guimarães AN, Ferraz L, Motta MGC. Cotidiano de famílias que convivem com o autismo infantil. Rev baiana enferm [Internet]. 2014 [cited 2019 Mar 20];28(3):271-82. Available from: file://C:/Users/Home/Downloads/1045138167-1-PB.pdf

23. González-Serna JMG, Ferreras-Mencia S, ArribasMarín JM. Desenvolvimento e validação da Escala Axiológica de Hospitalidade para Humanização da Assistência de Enfermagem. Rev latinoam enferm [Internet]. 2017 [cited 2019 Apr 20];25:e2919. Available from: http://www.scielo. br/pdf/rlae/v25/pt_0104-1169-rlae-25-e2919.pdf

24. Medeiros PF, Guareschi NMF. Políticas públicas de saúde da mulher: a integralidade em questão. Rev Estud Fem [Internet]. 2019 [cited 2019 Mar 20];17(1):31-48. Available from: http://www.scielo. br/pdf/ref/v17n1/a03v17n1.pdf

Recebido: 30 de maio de 2019 Aprovado: 26 de agosto de 2019 Publicado: 14 de novembro de 2019

A Revista Baiana de Enfermagem utiliza a Licença Creative Commons - Atribuição-NãoComercial 4.0 Internacional. https://creativecommons.org/licenses/by-nc/4.0/ Este artigo é de acesso aberto distribuído sob os termos da Licença Creative Commons (CC BY-NC). Esta licença permite que outros remixem, adaptem e criem a partir do seu trabalho para fins não comerciais. Embora os novos trabalhos tenham de lhe atribuir o devido crédito e não possam ser usados para fins comerciais, os usuários não têm de licenciar esses trabalhos derivados sob os mesmos termos. 